\title{
Compact $S$-band linear accelerator system for ultrafast, ultrahigh dose-rate radiotherapy
}

\author{
L. Faillace $\odot,{ }^{1,6}, *{ }^{*}$ S. Barone, ${ }^{2}$ G. Battistoni $\odot{ }^{3}$ M. Di Francesco, ${ }^{2}$ G. Felici $\odot,{ }^{2}$ L. Ficcadenti, ${ }^{4}$ \\ G. Franciosini, ${ }^{4,5}$ F. Galante, ${ }^{2}$ L. Giuliano $\odot,{ }^{1,4}$ L. Grasso, ${ }^{2}$ A. Mostacci, ${ }^{1,4}$ S. Muraro, ${ }^{3}$ M. Pacitti, ${ }^{2}$ \\ L. Palumbo, ${ }^{1,4}$ V. Patera $\odot,{ }^{1,4}$ and M. Migliorati ${ }^{1,4}$ \\ ${ }^{1}$ Sapienza University, Department of Basic and Applied Sciences for Engineering, \\ via A. Scarpa 14, 00161 Roma, Italy \\ ${ }^{2}$ S.I.T. Sordina IORT Technologies S.p.A., 04011 Aprilia, LT, Italy \\ ${ }^{3}$ INFN Sezione di Milano, via Celoria 16, 20133 Milano, Italy \\ ${ }^{4}$ INFN Sezione di Roma 1, Piazzale Aldo Moro 2, 00185 Rome, Italy \\ ${ }^{5}$ Sapienza University, Department of Physics, Piazzale Aldo Moro 2, 00185 Rome, Italy \\ ${ }^{6}$ INFN Laboratori Nazionali di Frascati, P.O. Box 13, I-00044, Frascati (Rome), Italy
}

(Received 17 December 2020; accepted 19 April 2021; published 17 May 2021)

\begin{abstract}
Radiation therapy is currently the most utilized technique for the treatment of tumors by means of ionizing radiation, such as electrons, protons and x/gamma rays, depending on the type, size and depth of the cancer mass. Radiation therapy has in general fulfilled the main requirement of targeting thus damaging the malignant cells and sparing the healthy tissues as best as possible. In this scenario, electron linear accelerators have been operated as viable tools for the delivery of both high-energetic electrons and $\mathrm{x}$-ray beams, which are obtained via the bremsstrahlung process of the electrons hitting on a high- $\mathrm{Z}$ material. Recently, it has been experimentally demonstrated that ultrahigh dose-rate bursts of electrons and $x$-ray beams increase the differential response between healthy and tumor tissues. This beneficial response is referred to as the FLASH effect. For this purpose, we have developed the first dedicated compact $S$-band linear accelerator for FLASH radiotherapy. This linac is optimized for a nominal energy of $7 \mathrm{MeV}$ and a pulsed electron beam current of $100 \mathrm{~mA}$ and above. The accelerator is mounted on a remote-controlled system for preclinical research studies in the FLASH regime. We will show the rf and beam dynamics design of the $S$-band linac as well as the commissioning and high-power rf tests. Furthermore, the results of the dosimetric measurements will be illustrated.
\end{abstract}

DOI: 10.1103/PhysRevAccelBeams.24.050102

\section{INTRODUCTION}

Over the past century, radiation therapy, usually referred to as radiotherapy (RT), has provided invaluable benefits for the treatment of tumors. Depending on the type, size and depth of cancer mass, different types of ionizing radiation, such as electrons, protons and x/gamma rays, are typically employed [1,2]. Today, RT is administered with high precision using intensity modulated RT, image guided RT, stereotactic body RT and proton therapy [3]. Dose fractionation and precise volume optimization in conventional RT [4] have enormously increased the normal tissue tolerance and opened the possibility to achieve a very high curative dose delivered to the tumor [5]. Typical delivered doses are

\footnotetext{
*Corresponding author. luigi.faillace@uniroma1.it

Published by the American Physical Society under the terms of the Creative Commons Attribution 4.0 International license. Further distribution of this work must maintain attribution to the author(s) and the published article's title, journal citation, and DOI.
}

around 2 Gy per fraction [6] per day and the average dose rate is of the order of $1 \mathrm{cGy} / \mathrm{s}$. Multiple treatment sessions for five days a week and several weeks are required so that the normal tissues and organs at risk have time to heal from the damaging effects of the ionizing radiation.

Recently, a new approach called FLASH radiotherapy (FLASH-RT) [7-11] has proved that shortening the overall time for the dose delivery, together with the above-mentioned advancements in conventional RT, could lead to the achievement of the increase of the normal tissue tolerance, thus allowing the delivery of higher curative doses, and the possibility to overcome the tumor radiation resistance.

The lowering of toxicity in healthy tissues, during the irradiation of mice, was observed for the first time by Hornsey and Alper in 1966 [12]. About a decade later, it was demonstrated by Hendry [13] that irradiating a mouse tail with very fast bursts of electrons with pulse lengths of $4.5 \mu \mathrm{s}$ induced resistance to epithelial necrosis which is characteristic of oxygen depletion at high dose rates. In 2014, a preclinical study was conducted at the Institut Curie [7] where ultrahigh dose-rate irradiation $(\geq 40 \mathrm{~Gy} / \mathrm{s}$, several orders of magnitude higher than those used in clinical 
practice today) was given in a single dose. The term FLASH was introduced in reference to ultrafast electron pulse delivery (few microseconds). In particular, the FLASH "effect" is defined by the biological effect. This experiment showed that FLASH irradiation protects lungs from radiation-induced fibrosis. In other words, FLASH was shown to be less fibrogenic than conventional irradiation. It results that the implementation of FLASH irradiation improves the differential response between healthy and tumor tissues. Therefore, this method is a crucial candidate as an alternative to conventional radiotherapy, with all its complications and drawbacks, but without any loss of antitumor efficiency [1420]. As a matter of fact, the first FLASH-RT clinical case was recently conducted at the Lausanne University Hospital [21]. The treatment was feasible and safe with a favorable outcome both on normal skin and the tumor. For this patient, indeed, the FLASH-RT reactions associated with the 15 Gy single dose delivery were minimal and disappeared in a much shorter time compared with dose fractionation in conventional-RT. Eventually, the tumor response appeared rapid and complete after only a 6-months follow-up.

The safety and performance requirements for a medical linear accelerator are particularly stringent. The International Standards [22-24] prescribe in details the precision associated to each particular feature, such as the output beam and energy stability, radiation protection, etc. For example, among the main specifications for the dose measurements, we recall here the short term repeatability, as defined, which must be better than $1 \%$, and the long term repeatability that must be better than $3 \%$.

Over the past half-century, radio-frequency (rf) linear accelerators (linacs) have provided high levels of reliability for conventional radiotherapy delivery. Recently, some existing linac systems have been modified [25-32] in order to operate at ultrahigh dose rates for reaching the FLASH regime which may represent a great promise for radiotherapy.

The first paper dealing with a "readapted" Flash linac can be found in [28]. Several other groups have tried such an approach with different linacs $[29,30]$ with the main features given in Table I. However, these initial experiments with modified existing linacs have shown at least two significant drawbacks: (i) The maximum field dimension achievable is generally smaller than $5 \times 5 \mathrm{~cm}$, and the field flatness is pretty poor; furthermore, the target must be placed in proximity to the linac exit. (ii) In order to achieve high dose per pulse the beam monitoring system is bypassed, therefore there is no real time control of the delivered beam.
As a consequence, it is clear that there are no existing modified linacs that could meet the European Norms (EN) standard requirements. Nevertheless, these machines showed either low-energy beams, which are inadequate for deep-seated tumors, or small achievable field sizes (e.g., diameter $<5 \mathrm{~cm}$ ). Moreover, there is the need to develop new standard dosimetry protocols [33-35] to bring the FLASH regime into clinical practice, since current state-ofthe-art dosimeters, such as ionization chambers, are precluded due to saturation phenomena.

In this paper, we propose the design and implementation of an ad hoc $S$-band linac system optimized to operate at ultrahigh dose rates for preclinical studies of the FLASH effect. The system will produce variable output field sizes, from $1 \mathrm{~cm}$ up to $10 \mathrm{~cm}$ diameter. We will refer to this design as the "ElectronFlash," hereinafter EF, which is the first dedicated research accelerator for FLASH radiation therapy (FLASH-RT). The system works in electron mode only, it is designed to produce both FLASH and conventional dose rates, with a nominal output electron beam energy of $7 \mathrm{MeV}$ with a pulsed current beyond $100 \mathrm{~mA}$. Therefore, with the new dedicated linac, it is possible to obtain a heavily pulsed beam, with dose rates that range from 0.01 up to $4000 \mathrm{~Gy} / \mathrm{s}$, and higher. This project effort is the result of a well-established collaboration between the University of Rome "Sapienza" and the private company SIT spa. We will discuss the rf and beam dynamics design and the highpower test measurements of the FLASH linac. Moreover, the dose calibration and monitoring for pulse to pulse stability, i.e., reliability of the linac-based machine, will be also presented. It has to be noted that the EF system will also work at lower energies than the nominal one as well as it will permit to increase the output beam energy by lowering the electron peak current. This collaboration effort aims at establishing the first step in the development of a reliable technology which can help define the FLASH effect in an accurate way. The EF system was commissioned by the Institut Curie, Orsay. The factory acceptance test was carried out at SIT and the system was installed at the Institut Curie in August 2020.

\section{LINAC RF AND BEAM DYNAMICS DESIGN}

The linac structure is today the key component for the production of electron or x-ray beams utilized in numerous applications from research, industry and medicine. Our FLASH linac system will follow the main features used in

TABLE I. Table of modified existing linacs.

\begin{tabular}{lcccc}
\hline \hline Linac type & $\begin{array}{c}\text { Beam } \\
\text { energy }\end{array}$ & $\begin{array}{c}\text { Average } \\
\text { dose rate }\end{array}$ & Max field size & $\begin{array}{c}\text { Beam } \\
\text { monitoring }\end{array}$ \\
\hline ELEKTA Precise [28] & $10 \mathrm{MeV}$ & $1000 \mathrm{~Gy} / \mathrm{s}$ & $<10 \mathrm{~cm} \times 10 \mathrm{~cm}$ & $\begin{array}{c}\text { Absent } \\
\text { Varian 2100 IX [29] }\end{array}$ \\
IORT NOVAC7 [30] & $90 \mathrm{MeV}$ & $33 \mathrm{~Gy} / \mathrm{s}$ & $<4 \mathrm{~cm} \times 4 \mathrm{~cm}$ & $\begin{array}{c}\text { Absent } \\
\text { Absent }\end{array}$ \\
\hline \hline
\end{tabular}


most medical and industrial linac-based machines, like the existing LIAC ${ }^{\mathrm{TM}}$ machine by SIT. The EF is thus an upgraded version of the LIAC for FLASH irradiation with electrons.

The high-power rf source is a commercial magnetron energized by a high-voltage modulator. The electron beam is generated by a thermionic electron gun and the linear accelerator is optimized in order to deliver the high-current electron beam. In this section, we will describe the rf and beam dynamics design of the electron gun and the $S$-band EF linac.

\section{A. The electron gun}

In Fig. 1 is shown the 2D model of the thermionic electron gun (e-gun) geometry used for the simulations with the code EGUN [36]. The working e-gun voltage is $12 \mathrm{kV}$. The anode aperture is chosen in order to confine the electron beam so to focus it to a waist into the first cell of the downstream linac. The equipotential lines from the simulations are given in green and the continuous flow of the electron beam is in blue. All units are in $0.1 \mathrm{~mm}$ scale. The current emitted by the cathode of the e-gun is $250 \mathrm{~mA}$, as per SIT's specifications. The emitting surface is about $4 \mathrm{~mm}$ in diameter. The simulation shows that the waist of the electron beam is about $1.5 \mathrm{~mm}$ in diameter, located about $1.3 \mathrm{~cm}$ from the cathode (indicated at 130 on the abscissa axis, where the angular divergence of the beam is zero).

\section{B. rf design of the FLASH linac}

The FLASH linac is a magnetically coupled biperiodic standing-wave (SW) accelerating cavity, operating in $S$-band $(2.998 \mathrm{GHz})$. The SW structure works in the $\pi / 2$ resonant mode with a high effective shunt impedance per unit length $\left(R_{s h}\right)$ and a high stability of the accelerating field configuration against external mechanical solicitations. The structure features a biperiodic geometry in which accelerating cells alternate with unexcited coupling cells.

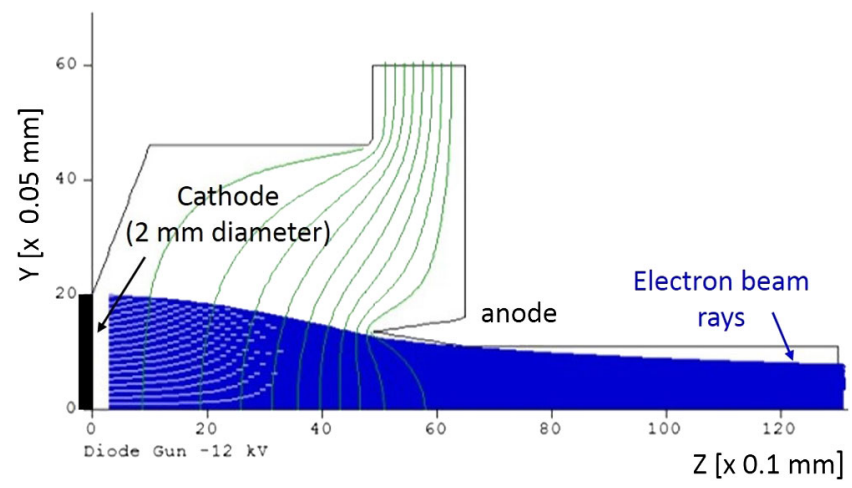

FIG. 1. 2D model of the electron gun. The equipotential lines from the simulations are given in green and the continuous flow of the electron beam is in blue. All units are in $0.1 \mathrm{~mm}$ scale.
All cells are attached to each other axially and are coupled via magnetic fields through coupling holes located off axis. The rf design of the linac was carried out with the CST MICROWAVE STUDIO SUITE code [37].

The linac rf design is optimized according to the main specifications on the output electron beam. The required nominal FLASH linac output beam energy is $7 \mathrm{MeV}$ with an electron beam peak current of at least $100 \mathrm{~mA}$.

In order to further increase the linac shunt impedance, the cells have a nose-cone type geometry. In Fig. 2 is given the sketch of a typical coupled cavity which was optimized and used in our project. The nose angle is $30^{\circ}$ and the cell length is equal to $\beta \lambda / 2$, where $\lambda$ is the rf field wavelength and $\beta=v / c$ is the electron velocity $v$ normalized to the speed of light $c$. The nose cone allows for the localization of very high electric field in the middle of the accelerating cells thus creating a very efficient acceleration of the beam propagating from the e-gun and along the linac axis. The nose-cone geometry allows for higher acceleration efficiency (i.e., higher effective shunt impedance), for a given input rf power.

The input $\mathrm{rf}$ power is provided by a commercially available $S$-band magnetron [38] which is able to deliver up to 3.1 MW of input power. The total number of cells is chosen as a compromise between the maximum output beam energy $(7 \mathrm{MeV})$, available rf power and linac compactness. The final choice of the number of the accelerating cells is 11 , resulting in a total length of $52 \mathrm{~cm}$. The bunching section is composed of the first five cells. Each single cell is designed to resonate at $f=2998 \mathrm{MHz}$, with an effective shunt impedance equal

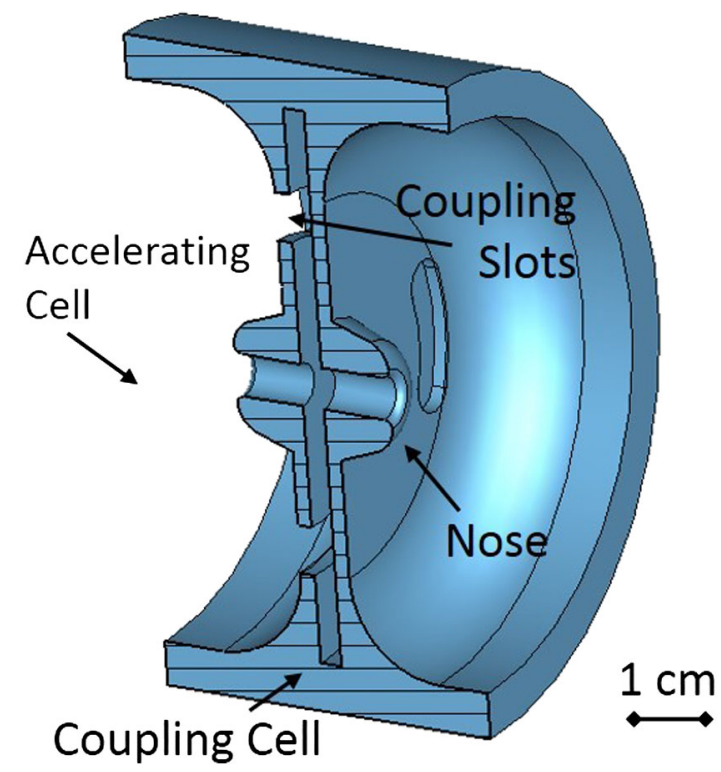

FIG. 2. Sketch of the $S$-band nose-cone cavity geometry. The nose-cone increases acceleration efficiency. The electromagnetic energy builds up in the accelerating cells through the coupling slots. 
TABLE II. Table of each cell main rf parameters.

\begin{tabular}{lccc}
\hline \hline Cell number & $f_{\text {res }}[\mathrm{GHz}]$ & $R_{\text {sh }}[\mathrm{M} \Omega / \mathrm{m}]$ & $\mathrm{Q}$ \\
\hline 1 & 2.990 & 53.5 & 10773 \\
2 & 2.987 & 91 & 13019 \\
3 & 2.990 & 93.3 & 14247 \\
4 & 2.991 & 91.6 & 14842 \\
5 & 2.991 & 90 & 15243 \\
6 & 2.994 & 90 & 14901 \\
7 & 2.991 & 90 & 15243 \\
8 & 2.991 & 90 & 15243 \\
9 & 2.991 & 90 & 15243 \\
10 & 2.991 & 90 & 15243 \\
11 & 2.993 & 94 & 15887 \\
\hline \hline
\end{tabular}

to $R_{s h}=90 \mathrm{M} \Omega / \mathrm{m}$ and a quality factor $Q$ in the range (13000-15000) as shown in Table II.

The 3D rendering of the coupled-cavity linac is shown in Fig. 3. In the figure is also included the input rf waveguide (WR284) which delivers the high power to linac from the magnetron and is matched to the cavity through a dedicated tapered section.

The electric field vector plot of the $\pi / 2$ operation mode is shown in Fig. 4. For the total linac we have obtained a resonant frequency equal to $f_{\pi / 2}=3000 \mathrm{MHz}$, well within the magnetron bandwidth, an average quality factor $Q=14900$ and average effective shunt impedance equal to $R_{s h}=90 \mathrm{M} \Omega / \mathrm{m}$.

In Fig. 5 is shown the on-axis accelerating electric field. The field distribution shows a good flatness.

On the second-last cell, a magnetic loop is inserted in order to perform on-line measurements of the rf signal, such as field electromagnetic field buildup inside the linac. The CAD model which shows the magnetic loop insertion

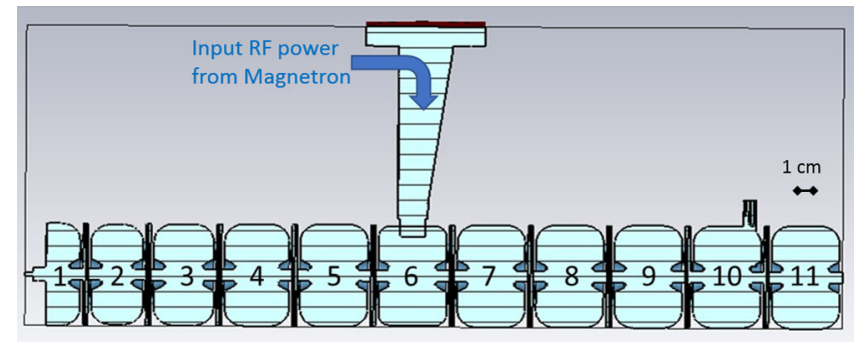

FIG. 3. 3D rendering of the coupled-cavity linac. The input rf waveguide (WR284) is matched to the linac through a tapered section.

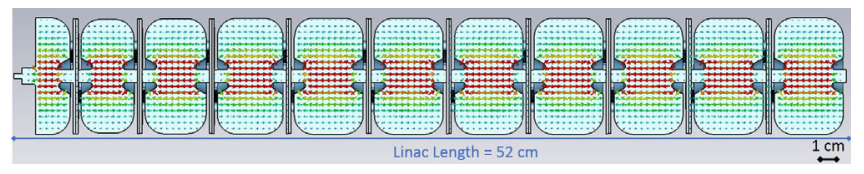

FIG. 4. Electric field vector plot of the $\pi / 2$ operation mode.

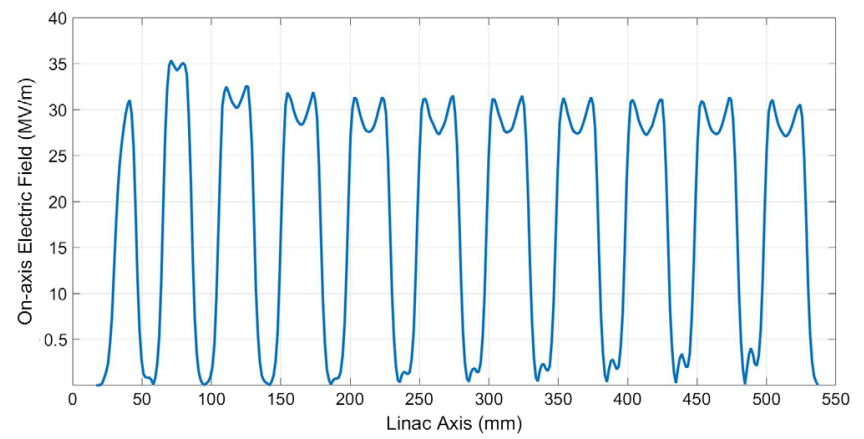

FIG. 5. On-axis accelerating electric field.

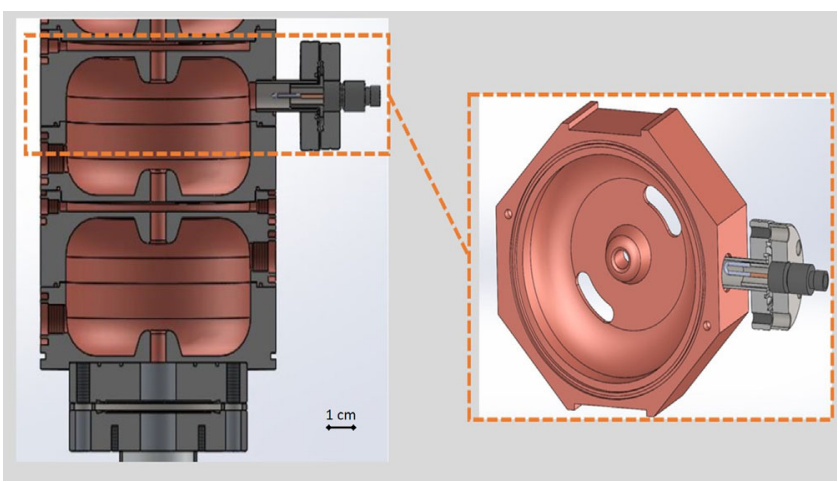

FIG. 6. Cavity with magnet loop for on-line measurements of the rf signal.

is given in Fig. 6. After inserting the magnetic loop in the cell, the diameter of this cell was modified in order to keep the design resonant frequency and the desired flatness of the electric field.

\section{Optimization of waveguide-to-linac rf power matching}

In order to obtain efficient coupling between the magnetron and the accelerating structure, the rf waveguide is inserted in the middle cell (coupler cell). Central coupling allows to get rid of half of the linac resonant modes, which are then not excited. The waveguide-to-linac coupling coefficient $\beta_{c}$ must be optimized to minimize the reflected rf power when the electron beam is accelerated. The formula we used as reference is the following [39]:

$$
\beta_{c}=1+\frac{P_{b}}{P_{d}}=1+\frac{I_{p} R_{s h} l}{V_{a c c}},
$$

where $P_{b}$ is the beam power, $P_{d}$ is the power dissipated in the cavity walls, $I_{p}$ is the beam peak current, $R_{s h}$ is the effective shunt impedance, $l$ is the linac length, and $V_{a c c}$ is the accelerating voltage. Taking into account the rf linac parameters, as illustrated above, we have obtained the optimal value to be $\beta_{c}=1.52$. In order to achieve this required coupling coefficient, various tuning steps of the 


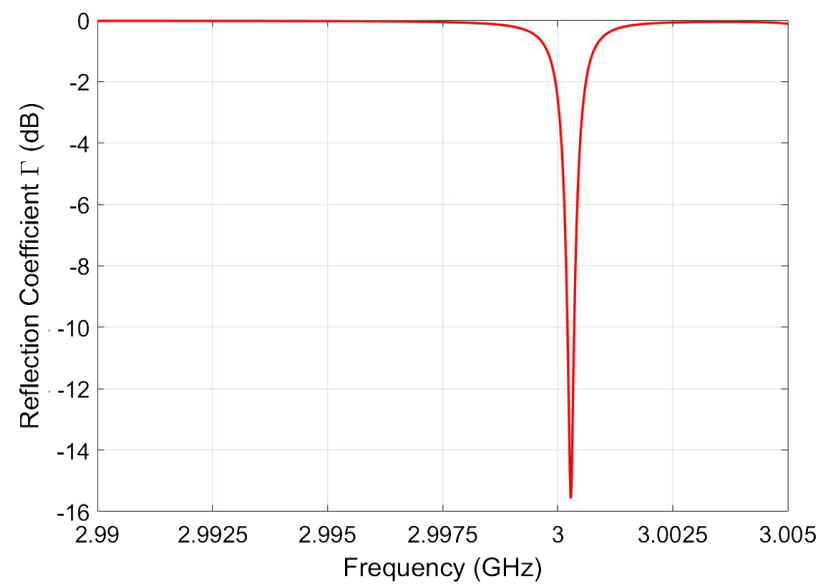

FIG. 7. Linac reflection coefficient $\Gamma$.

coupling slot dimensions and geometry of the coupler cell were performed. The linac reflection coefficient, $\Gamma$, is plotted in Fig. 7 as a function of frequency. The resonant $\pi / 2$ mode is located at $f_{\pi / 2} \sim 3 \mathrm{GHz}$ with a reflection coefficient of $\Gamma=-13.8 \mathrm{~dB}(\sim 0.2$ in linear scale), which confirms the required coupling coefficient, since $\beta_{c}=$ $(1+\Gamma) /(1-\Gamma)$. The plot also shows that only one resonant mode is excited inside the structure within the working bandwidth of the magnetron. This reassures that no other electromagnetic field can interfere with the operational one.

In Table III, we list the optimized linac main rf parameters. The required input rf power is about $2 \mathrm{MW}$ in order to achieve the nominal operation requirements, i.e., electron beam energy of $7 \mathrm{MeV}$ and current of $100 \mathrm{~mA}$.

\section{Beam dynamics simulations}

The beam dynamics (BD) simulations were carried out with the 2D numerical code T-STEP [40]. The input electron beam distribution was imported from the EGUN output. The $\mathrm{BD}$ performance is optimized to achieve three main requirements; electron beam energy of $7 \mathrm{MeV}$, electron beam peak current above $100 \mathrm{~mA}$; variable beam transverse size (up to $10 \mathrm{~cm}$ diameter) at the target located at $80 \mathrm{~cm}$ from the linac exit.

The BD performance was optimized in parallel with the rf design. The cell lengths, in particular for the initial

TABLE III. Output electron beam parameters.

\begin{tabular}{lc}
\hline \hline Parameter & Value \\
\hline Frequency & $2.998 \mathrm{GHz}$ \\
Effective shunt impedance, $R_{s h}$ & $90 \mathrm{M} \Omega / \mathrm{m}$ \\
Quality factor, $Q$ & 14,000 \\
Cell-to-cell coupling coefficient, $K$ & $3 \%$ \\
Waveguide-to-linac coupling coefficient, $\beta_{c}$ & 1.52 \\
rf power consumption @ 7 MeV & $2 \mathrm{MW}$ \\
Linac length & $52 \mathrm{~cm}$ \\
\hline \hline
\end{tabular}

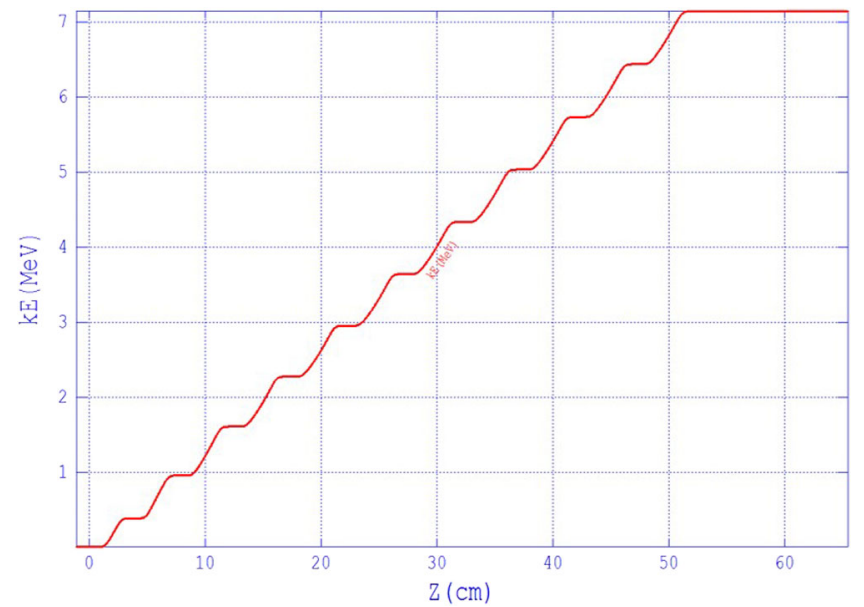

FIG. 8. Beam energy gain from $z=0 \mathrm{~cm}$ (linac input) to $z=67 \mathrm{~cm}$ (linac output). The final energy is $7 \mathrm{MeV}$.

bunching section, were chosen in order to obtain acceleration synchronism between the low-energy beam and the accelerating electric field from the e-gun all the way through the linac. In Fig. 8 is shown the beam energy gain from $z=0 \mathrm{~cm}$ (linac input) to $z=67 \mathrm{~cm}$ (linac output). The final energy is $7 \mathrm{MeV}$.

The electron beam phase-space projections at the linac entrance are illustrated in Fig. 9. The beam spot diameter is about $1.5 \mathrm{~mm}$ and its length is equal to one wavelength of the operating nominal frequency $(2.998 \mathrm{GHz})$. The beam is basically monoenergetic with the energy $E=12 \mathrm{keV}$ from the electron gun. The energy spread $\Delta E=E-E_{s}$, where $E_{s}$ is the reference particle energy, is negligible, as shown in Fig. 9(d).

The final BD optimization led to the required output beam parameters, given in Fig. 10. The beam output peak current reaches the value of $105 \mathrm{~mA}$, corresponding to a
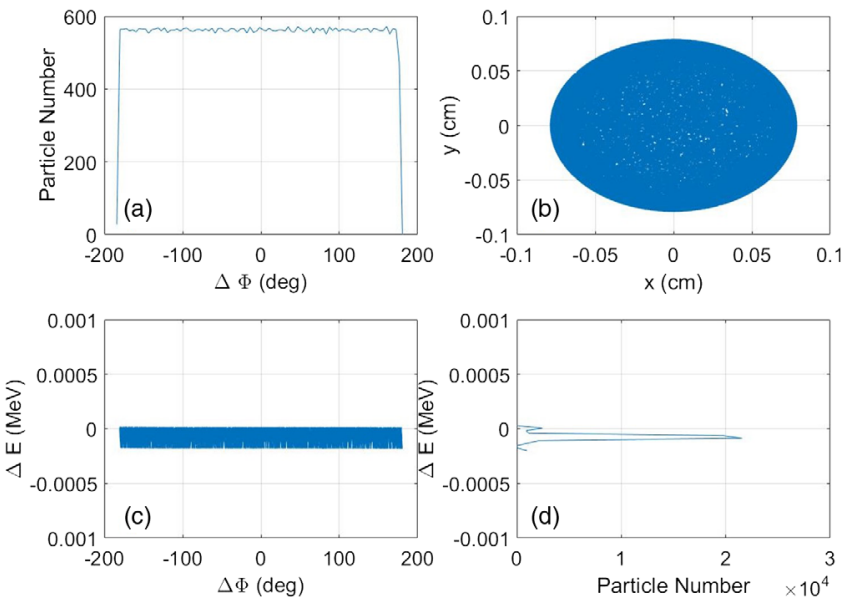

FIG. 9. T-Step input electron beam parameters. (a) longitudinal phase distribution in degrees; (b) beam transverse spot size in cm; (c) beam longitudinal phase-space (energy vs phase); (d) beam energy spectrum in $\mathrm{MeV}$. 

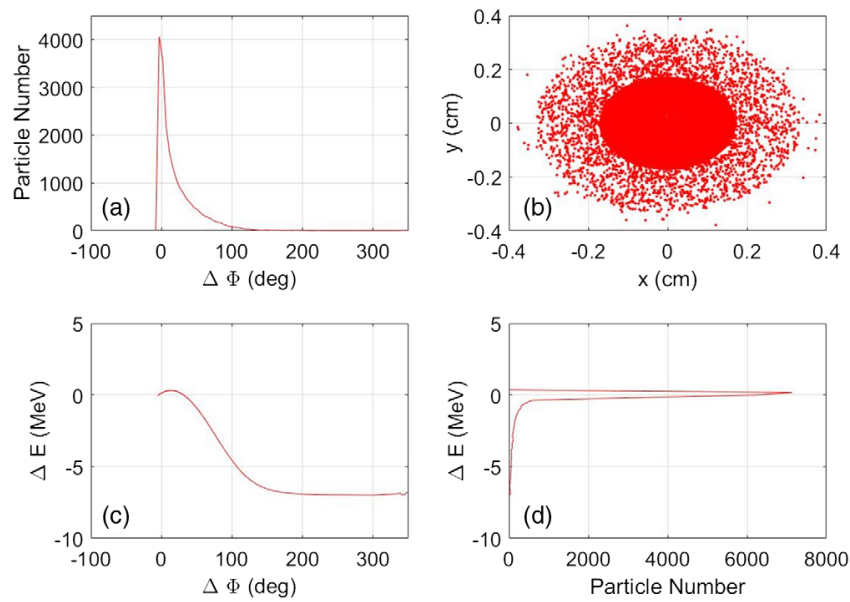

FIG. 10. T-STEP output electron beam parameters. (a) Longitudinal phase distribution in degrees; (b) beam transverse spot size in cm; (c) beam longitudinal phase-space (energy vs phase);

(d) beam energy spectrum in $\mathrm{MeV}$.

beam capture of $42 \%$ of the input e-gun beam, assumed to be $250 \mathrm{~mA}$. The transverse beam size is $6 \mathrm{~mm}$ in diameter at the linac exit. The beam energy spread shows a FWHM value below $10 \%$. The beam output nominal parameters are summarized in Table IV.

In order to fulfill the requirement on the adjustability of the beam transverse size downstream the linac, we have studied two technological approaches. The first one is active and employs quadrupole magnets with variable focusing/ defocusing gradients for beam spot manipulation. The other one is passive and makes use of hollow cylindrical applicators of polymethylmethacrylate (PMMA) with different diameters. In this section, we describe the first method used in beam dynamics simulations. The second method is used in dose simulations, as discussed in Sec. III. For the measurements, we have used both methods but eventually decided to use the PMMA applicators (see Sec. VI) for the EF system.

For the beam dynamics simulations of the FLASH linac, two quadrupole magnets $(Q 1$ and $Q 2)$ are employed. A quadrupole magnet will focus the electron beam in one direction and defocus it in the other direction. Each magnet's location, gradient, bore radius and effective length are optimized with T-STEP and the TRACE3D code [41]. By properly choosing these parameters, it is possible to deliver

TABLE IV. Output electron beam parameters.

\begin{tabular}{lc}
\hline \hline Parameter & Value \\
\hline Frequency & $2.998 \mathrm{GHz}$ \\
Output beam energy & $7 \mathrm{MeV}$ \\
Output beam current & $100 \mathrm{~mA}$ \\
Beam diameter & $6 \mathrm{~mm}$ \\
Beam capture & $>40 \%$ \\
Beam energy spread & $<10 \%$ \\
\hline \hline
\end{tabular}

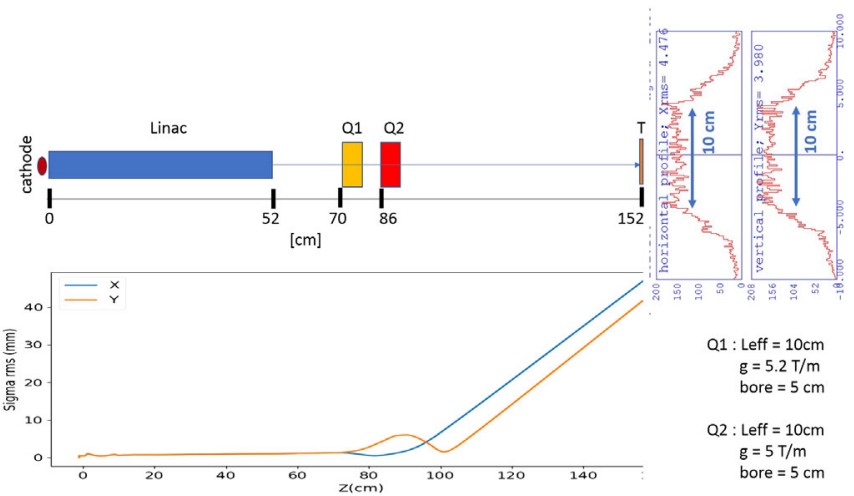

FIG. 11. Sketch of the accelerator system (e-gun and linac) with the quadrupole magnets $(\mathrm{Q} 1$ and Q2). The electron beam transverse distribution at $80 \mathrm{~cm}$ from the linac exit is also shown.

an electron beam with a variable transverse spot size after the linac. In Fig. 11 (top), we show the sketch of the accelerator system (e-gun and linac) with the quadrupole magnets. In the same figure (bottom), the beam transverse profile evolution is plotted along the $\mathrm{x}$ direction (in blue) and the $y$ direction (in orange). The beam reaches a spot size of $10 \mathrm{~cm}$ in both directions at about $80 \mathrm{~cm}$ from the linac exit. The beam spot can be easily varied by modifying the magnets' gradients, for example. The nominal gradients for the two quadrupole magnets are $g_{1}=5.2$ and $g_{2}=5 \mathrm{~T} / \mathrm{m}$, respectively. The main magnets' parameters are listed in Table V.

\section{DOSE SIMULATIONS}

The numerical code FLUKA was used to implement dosimetric simulations in order to calculate the delivered dose with the electron bunch generated from the BD simulations. The current pulse, used as input to FLUKA, contains a limited number of $10^{8}$ electrons, for computing capacity purposes, with a $7 \mathrm{MeV}$ end energy. The electron distribution is rectangular with a diameter of $5 \mathrm{~mm}$. The electrons and photons with energy $<10 \mathrm{keV}$ are not transported and generated since they deposit their energy locally. Figure 12 shows a sketch of the simulation model. The linac exit is closed with a $60 \mu \mathrm{m}$ titanium window which seals the vacuum. A PMMA applicator is directly attached to the linac exit and is in air. The applicator diameter is $10 \mathrm{~cm}$. The target, a $5 \times 5 \times 5 \mathrm{~cm}^{3}$ water phantom is located $100 \mathrm{~cm}$ downstream the linac.

A two-dimensional projection of the dose spatial distribution in the water phantom is shown in Fig. 13.

TABLE V. Magnetic quadrupoles' nominal parameters.

\begin{tabular}{lc}
\hline \hline Parameter & Value \\
\hline Gradient & $5.2 \mathrm{~T} / \mathrm{m}(\mathrm{Q} 1), 5 \mathrm{~T} / \mathrm{m}(\mathrm{Q} 2)$ \\
Effective length & $10 \mathrm{~cm}$ \\
Bore diameter & $5 \mathrm{~cm}$ \\
\hline \hline
\end{tabular}




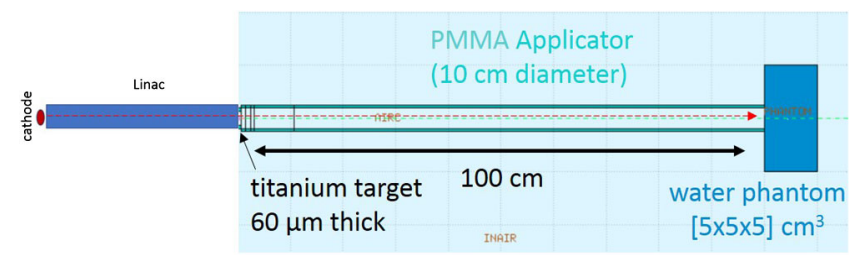

FIG. 12. Sketch of the dose simulation model with FLUKA. A PMMA applicator (with $10 \mathrm{~cm}$ diameter) is directly attached to the linac exit and is in air. The target, a $5 \times 5 \times 5 \mathrm{~cm}^{3}$ water phantom, is located at $100 \mathrm{~cm}$ from the linac.

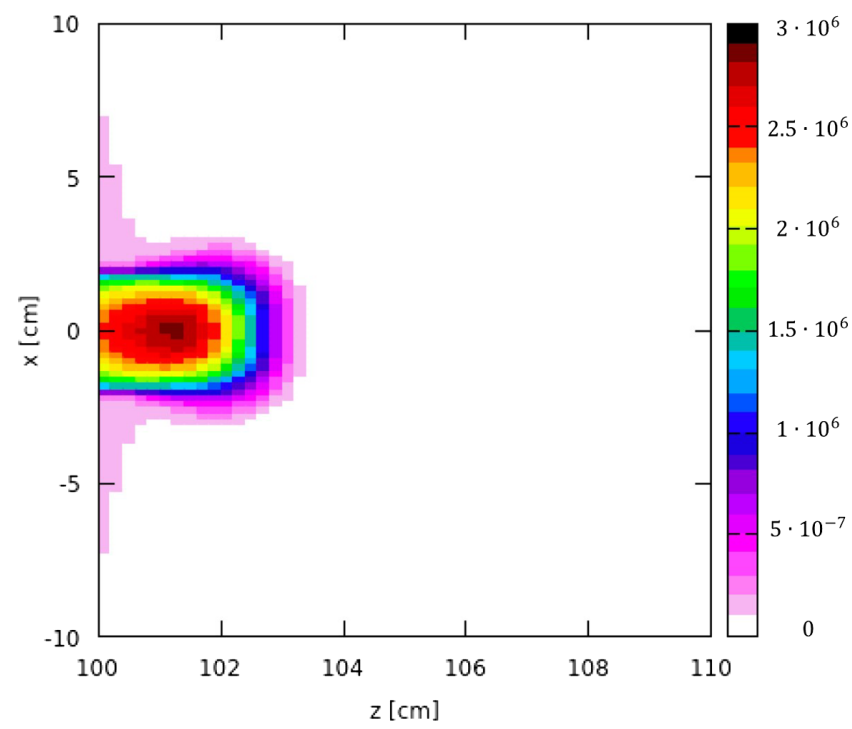

FIG. 13. Two-dimensional projection of the dose map in the water phantom for the $7 \mathrm{MeV}$ electron beam. Dose values are plotted using a linear color scale. Units are $\mathrm{GeV} / \mathrm{g}$ per primary electron.

The single electron released dose results to be $D=$ $5.28 \times 10^{-13} \mathrm{~Gy} /$ electron with a maximum value of $D_{\max }=2.14 \times 10^{-12} \mathrm{~Gy} /$ electron. In the simulation, the dose is calculated over an active volume with a transverse area of $2 \times 2 \mathrm{~mm}^{2}$ and a thickness of $10 \mathrm{~cm}$.

In order to evaluate the dose release of the electron beam obtained from the T-STEP code, the initial FLUKA results are then scaled up to an equivalent beam current of $I=100 \mathrm{~mA}$ with a pulse length equal to $t=4 \mu \mathrm{s}$ (corresponding to $2.5 \times 10^{12}$ electrons). The estimation gives a dose per pulse equal to $D_{p}=4.9$ Gy which corresponds to an instantaneous dose rate of $\dot{D}=1.22 \times 10^{6} \mathrm{~Gy} / \mathrm{s}$. The average dose rate, assuming a repetition rate of $250 \mathrm{~Hz}$, results to be $\langle\dot{D}\rangle=1.22 \times 10^{3} \mathrm{~Gy} / \mathrm{s}$, which confirms operation well beyond the minimum requirements for FLASH regime.

\section{LINAC LOW-POWER RF TESTS}

After the final engineering, the FLASH linac was handed over to SIT in order to proceed to the manufacturing, tuning, low-power rf characterization and high-power

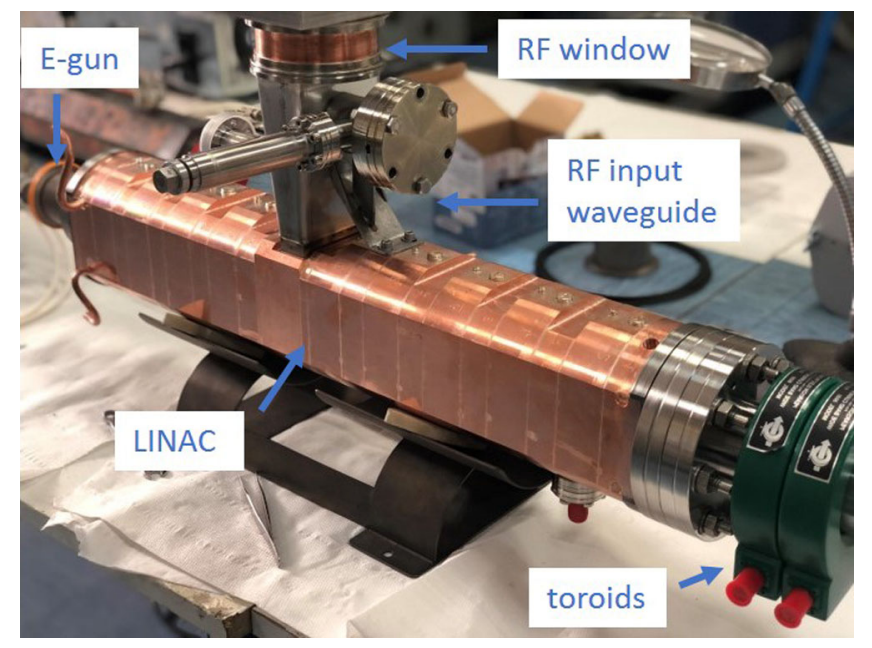

FIG. 14. Linac after brazing. The electron gun and the rf window are welded onto the structure. Two magnetic toroids (in green) for beam current monitoring are located on the output beampipe.

commissioning. The linac after final brazing is shown in Fig. 14. The electron gun and the rf window are welded onto the structure. Two magnetic toroids for beam current monitoring are located around the output beampipe.

In this section, we show the results of the linac tuning and low-power rf characterization. The tuning system consists of copper screws located at the equator of each accelerating and coupling cell. The tuning procedure adopts a plunger-type methodology which was achieved by means of two metallic rods, one on each side of the linac. The tuning algorithm is an iterative process. First, each accelerating cell is tuned to the specified resonant frequency of the $\pi / 2$ operating mode. Then, all coupling cells are tuned to suppress all unwanted modes at the same frequency.

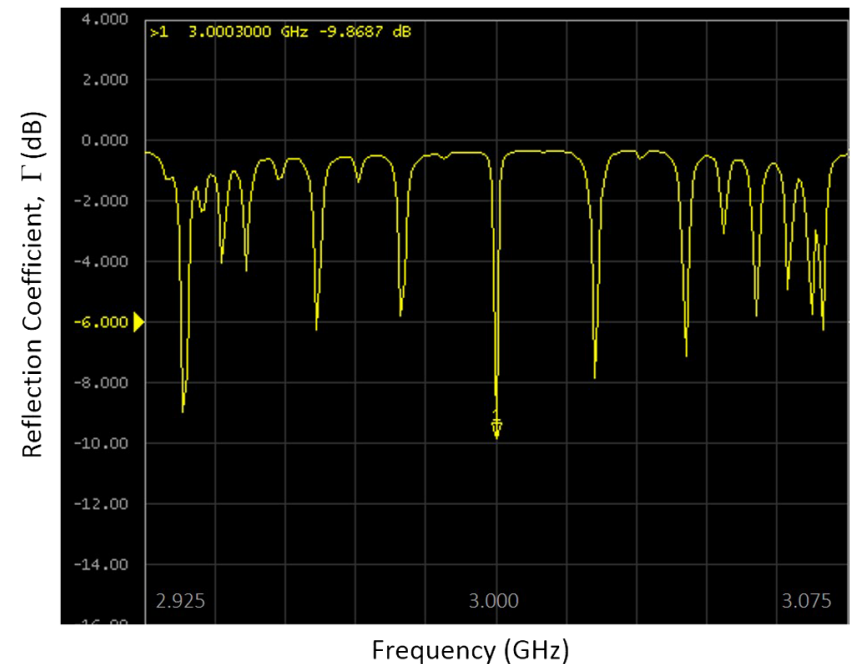

FIG. 15. Reflection coefficient $\Gamma$ in $\mathrm{dB}$ of the FLASH linac from the vector network analyzer. The frequency scale is $15 \mathrm{MHz}$ per division. 


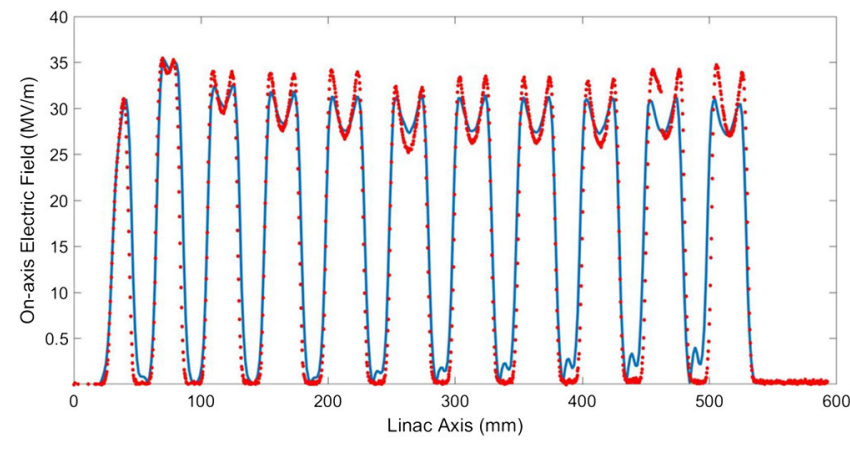

FIG. 16. On-axis electric field. The measured field profile (solid blue line) measured by means of the bead-pull technique shows good agreement with the field simulated with CST (dotted red line).

The algorithm basically predicts the depth of each single screw inside the cavity walls in order to minimize the reflection coefficient as well as to rid the linac of all unwanted modes. In Fig. 15 is shown the linac reflection coefficient $\Gamma$, acquired from the vector network analyzer, after the final tuning. The plot shows the operating $\pi / 2$ mode resonating at $3000.225 \mathrm{MHz}$ (in air) and a nearestmodes separation of $\pm 20 \mathrm{MHz}$.

The on-axis accelerating electric field was measured with the nonresonant bead-pull technique. In Fig. 16, we show the plot of the measured electric field along the linac axis together with the simulated one. The comparison shows perfect agreement between the measurements and the rf simulations.

\section{LINAC COMMISSIONING AND HIGH-POWER RF TESTS}

After the low-power rf characterization, the FLASH linac was mounted inside the mechanical envelope and the whole system, the ElectronFlash, was robotized and ready for commissioning. A picture of the $\mathrm{EF}$ rendering is given in Fig. 17. The EF was installed inside one of the bunkers available at SIT. The dose rate was varied

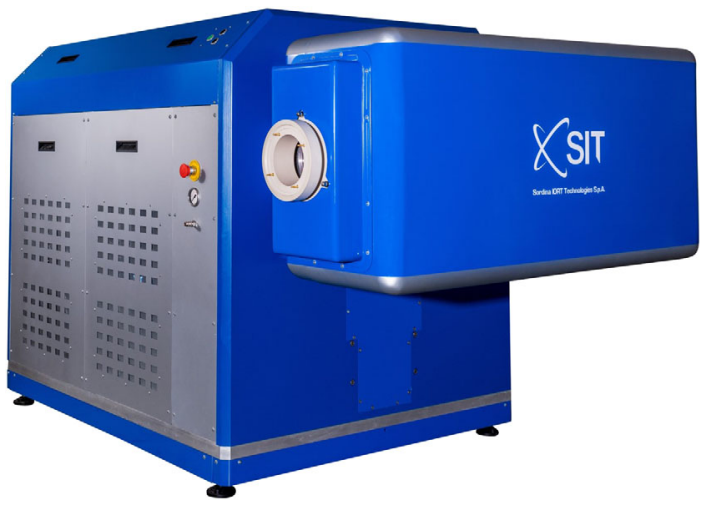

FIG. 17. Photograph of the ElectronFlash robotized system. The FLASH linac is located inside the movable irradiation head.

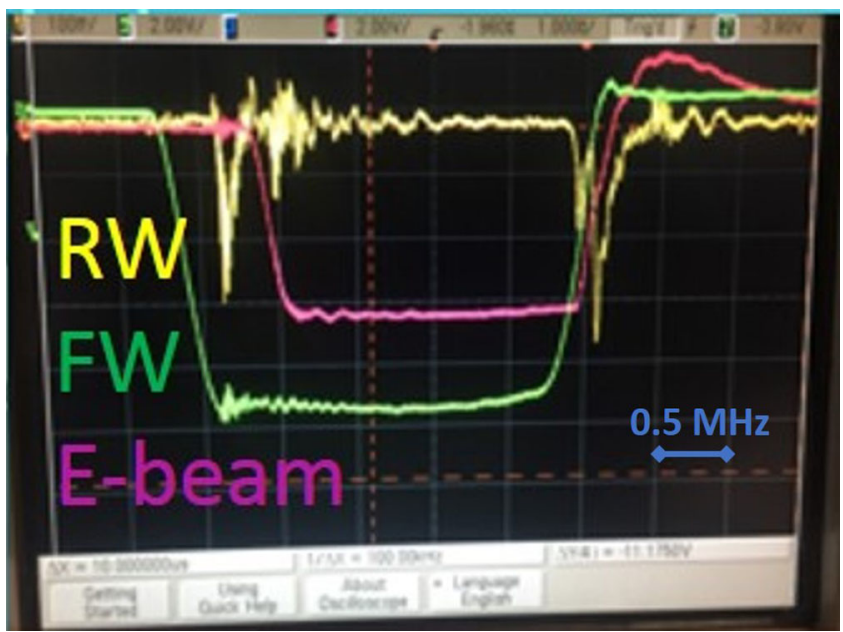

FIG. 18. The oscilloscope traces of the two monitored signals; the forward rf power (FW in green) from the magnetron and the reverse or reflected power (RW in yellow) from the linac. The beam current signal measured through the toroids at the linac exit is also displayed, in purple.

according to the following settings: pulse repetition frequency (PRF), which can be set in the range from $1 \mathrm{~Hz}$ up to $350 \mathrm{~Hz}$ with $1 \mathrm{~Hz}$ increments; e-gun peak current and e-gun pulse width $(\Delta \mathrm{t})$, which varies in a wide range (from 0.5 to $4 \mu \mathrm{s})$.

The high-power rf signals were monitored by means of a $60 \mathrm{~dB}$ directional coupler located before the input waveguide to the linac. The oscilloscope traces of the two monitored signals are shown in Fig. 18; the forward rf power $(\mathrm{FW})$ from the magnetron and the reverse or reflected power (RW) from the linac. On the same screen, the beam current signal measured through the toroids at the linac exit is also displayed. The input rf pulse has a flattop shape with a length of about $4 \mu \mathrm{s}$. The rise and drop times are about $0.5 \mu \mathrm{s}$ each. The electron bunch is injected into the linac with a delay of about $1 \mu \mathrm{s}$ in order to locate the flat part of the rf pulse. From the traces, it can be noted the perfect matching, i.e., zero reflected power, between the input rf signal and the electron beam.

\section{DOSE MEASUREMENTS}

At the output of the EF radiant head it has been implemented a dual dose monitoring system. When EF works in conventional mode, the dosimetric output is monitored by means of two ionization chambers inserted along the beam axis. Otherwise in FLASH mode the ionization chambers are not adequate for beam monitoring, due to the saturation problem that occurs at very high doseper-pulse values $[30,34,42,43]$. Therefore, in FLASH mode they are automatically moved outside the beam line and the dose monitoring system is composed by two toroidal inductors. Thanks to this dual dose monitoring system, 
an excellent signal-to-noise ratio is obtained in both irradiation modes.

We acquired and installed the two quadrupole magnets, discussed in Sec. II D, in order to test the beam field size variability and collimation. Nevertheless, we eventually decided to use our previously and well-established passive beam collimation system for the dose measurements shown in this paper. This system consists of a set of polymethylmethacrylate (PMMA) cylindrical applicators that can be directly attached to the radiant head. The internal diameters range from $\phi=10 \mathrm{~mm}$ up to $\phi=120 \mathrm{~mm}$, with $5 \mathrm{~mm}$ wall thickness. The source surface distance (SSD) varies according to the size of the applicator diameter, in order to guarantee specific values of beam flatness and symmetry.

In the ultrahigh dose-rate region, or FLASH mode, the dosimetric characterization cannot be performed using ionization chambers [35]. For this reason, during the commissioning, the dosimetric measurements were performed by means of radiochromic films (GAFCHROMIC EBT-XD, by Ashland) [44]. Radiochromic films are independent dose-rate dosimeters and provide high spatial resolution [45]. Therefore, they allow an extensive dosimetric characterization of the e-beam but they do not provide online dosimetric information such as ionization chambers. The dosimetry system includes films, a flat-bed scanner and a dedicated analysis software (Film QA Pro) [46].

During the commissioning, relative and absolute dose measurements were performed both in FLASH and conventional mode. For the acceptance procedure both the shortterm and the long-term energy stability has been evaluated. For the long-term stability three depth-dose measurements with the reference applicator $(100 \mathrm{~mm}$ diameter and $\mathrm{SSD}=93 \mathrm{~cm}$ ) were performed on three consecutive days. The ElectronFlash appeared to be very stable in terms of day-to-day energy stability.

For the experimental setup was used a RW3 solid phantom (water equivalent). The radiochromic film was placed inside the RW3 phantom, between two different slabs and parallel to the beam axis. The e-gun pulse duration $\Delta \mathrm{t}$ is equal to $4 \mu \mathrm{s}$. The irradiations were performed at 5 and $7 \mathrm{MeV}$, both in FLASH and conventional mode. The depth-dose curves (PDD) for a 5 and $7 \mathrm{MeV}$ e-beams are shown in Fig. 19.

For a $7 \mathrm{MeV}$ irradiation in FLASH mode, EF reached a dose per pulse $\left(D_{p}\right)$ with the reference applicator $(\phi=$ $100 \mathrm{~mm}$ ) equal to $5 \mathrm{~Gy} / \mathrm{p}$, furthermore this value rises up to $30 \mathrm{~Gy} / \mathrm{p}$ with the $1 \mathrm{~cm}$ applicator.

As discussed above, the PRF can be set in the range from 1 up to $350 \mathrm{~Hz}$. However, due to solid state modulator limits, the maximum power is limited at $250 \mathrm{~Hz}$, hence the maximum achievable dose rate is given by

$$
D R_{\max }=250 \cdot D_{p}
$$

Therefore, in this configuration we fully exceed the lower limit of $40 \mathrm{~Gy} / \mathrm{s}$ proposed for the FLASH region.

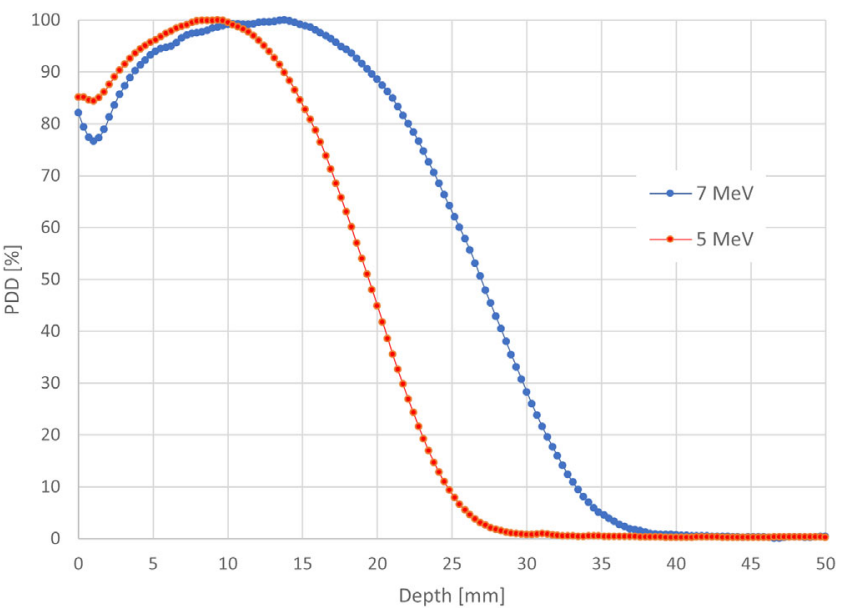

FIG. 19. Depth-dose curves for a $5 \mathrm{MeV}$ (red) and $7 \mathrm{MeV}$ (blue) e-beam measured in RW3 solid phantom with EBT-XD.

Indeed, setting the PRF to $250 \mathrm{~Hz}$ EF reached an average dose rate of $1200 \mathrm{~Gy} / \mathrm{s}$ with the reference applicator. The average dose-rate value increases with smaller diameter applicators, up to almost $7500 \mathrm{~Gy} / \mathrm{s}$ with the $1 \mathrm{~cm}$ applicator. The ratio between dose per pulse and pulse length gives the dose rate within pulse, or instantaneous dose rate. For these measures it was considered a pulse length of $4 \mu \mathrm{s}$, so it was obtained an instantaneous dose rate of $1.2 \times 10^{6} \mathrm{~Gy} / \mathrm{s}$ with the reference applicator. Considering the $1 \mathrm{~cm}$ applicator this value increases to almost $7.5 \times 10^{6} \mathrm{~Gy} / \mathrm{s}$.

Figure 20 shows the good agreement in the comparison between measurements and simulations of the PDD for the $7 \mathrm{MeV}$ case with the PMMA reference applicator

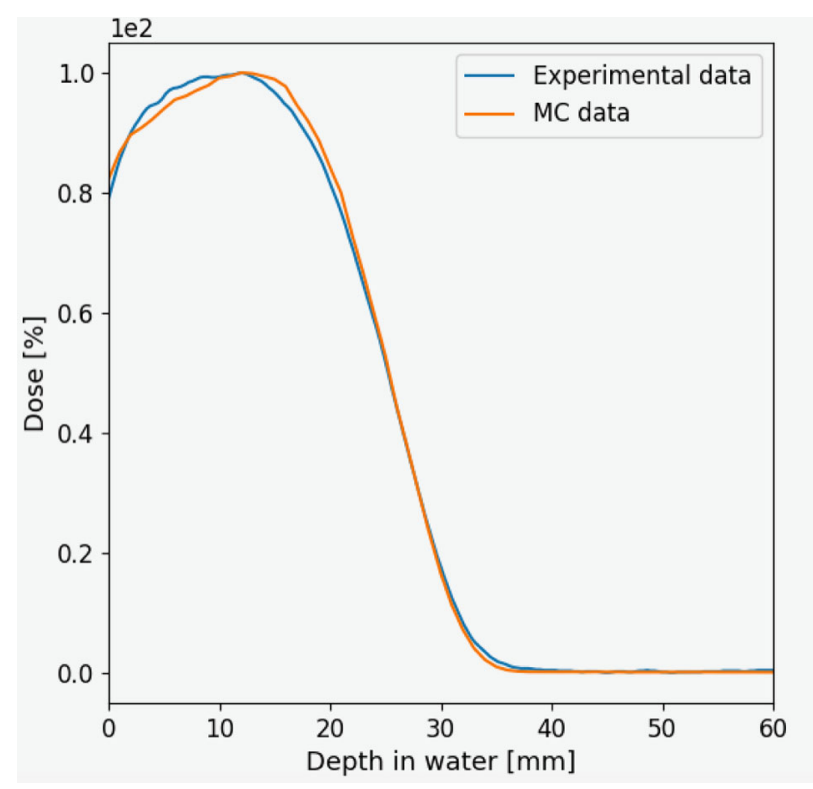

FIG. 20. Depth-dose curves for the $7 \mathrm{MeV}$ electron beam; simulation data (red) and experimental data (blue). 
TABLE VI. Output electron beam parameters.

\begin{tabular}{lcc}
\hline \hline Parameter & Simulation & Measurement \\
\hline Electron energy & $7 \mathrm{MeV}$ & $7 \mathrm{MeV}$ \\
Beam pulse length & $4 \mu \mathrm{s}$ & $4 \mu \mathrm{s}$ \\
Pulse current & $100 \mathrm{~mA}$ & $100 \mathrm{~mA}$ \\
Rep rate & $250 \mathrm{~Hz}$ & $250 \mathrm{~Hz}$ \\
Dose per pulse, $D_{p}$ & $4.9 \mathrm{~Gy}$ & $5.14 \mathrm{~Gy}$ \\
In-pulse dose rate, $\dot{D}$ & $1.22 \times 10^{6} \mathrm{~Gy} / \mathrm{s}$ & $1.28 \times 10^{6} \mathrm{~Gy} / \mathrm{s}$ \\
Average dose rate, $\langle\dot{D}\rangle$ & $1.22 \times 10^{3} \mathrm{~Gy} / \mathrm{s}$ & $1.28 \times 10^{3} \mathrm{~Gy} / \mathrm{s}$ \\
\hline \hline
\end{tabular}

(10 $\mathrm{cm}$ diameter). The main dose parameters are reported in Table VI where the electron pulse length is about $4 \mu$ s with a $250 \mathrm{~Hz}$ repetition rate. The measured average dose rate $\langle\dot{D}\rangle=1.28 \times 10^{3} \mathrm{~Gy} / \mathrm{s}$ confirms the achievement of the FLASH regime. The agreement between simulations and measurements also validates our rf, beam dynamics and dosimetric models.

The standard request for medical linacs is to keep long term stability and short term stability below 3\% and 1\% respectively [22], as discussed in Sec. I. Unluckily, the intrinsic accuracy of dose measurement was not better than $5 \%$ (due to intrinsic limitation of the radiochromic dose measurement). Nevertheless, the behavior of the beam monitoring system seems very promising and, once a better accuracy on the dose measurement is achieved [35], we are confident such threshold will be met.

\section{CONCLUSIONS}

The use of ultrahigh dose electron bursts, FLASH radiotherapy, has proved to provide unprecedented benefits for the treatment of tumors, compared to conventional radiotherapy. It has indeed been verified, under biological observation, a reduction of radiation toxicity to normal, healthy tissues, while maintaining a similar effect on tumors. This biological result has been referred to as the FLASH effect.

Various existing linac systems have been modified in order to operate in FLASH regime. Nevertheless, these machines showed either low-energy beams, which are inadequate for deep-seated tumors, or small achievable field sizes.

In this paper, we have developed the design, realization, commissioning and dosimetric characterization of the first dedicated $S$-band linac system optimized to operate at ultrahigh dose rates in FLASH regime and to produce variable output field sizes (up to $12 \mathrm{~cm}$ diameter during the dose measurements). The linac-based robotized system, called the "ElectronFlash" is then the first dedicated research accelerator for FLASH radiation therapy. This project effort is the result of a well-established collaboration between the University of Rome "Sapienza" and the private company SIT spa. This collaboration effort aims at establishing the first step in the development of a reliable technology which can help define the FLASH effect in an accurate way. The EF system is currently installed at the Institut Curie, Orsay.

The system is optimized to operate in electron modality with two nominal output beam energies: 5 and $7 \mathrm{MeV}$ with a pulsed current beyond $100 \mathrm{~mA}$. The collimation system for the here presented measurements consisted in the utilization of PMMA applicators with different diameters. Nevertheless, a dual magnetic quadrupole scheme was optimized and patented. The dose measurements have confirmed the production of high-current beams with dose rates that range from 1,200 up to $10,000 \mathrm{~Gy} / \mathrm{s}$ in the case of the 10 and $1 \mathrm{~cm}$ applicator, respectively. These dose rates are well beyond the threshold required for the FLASH effect.

Another innovative feature of the ElectronFlash is the possibility of on-line dose monitoring in dual-mode operation; conventional mode, through ionization chambers, and FLASH mode, by means of purposely calibrated magnetic toroids for electron beam current readings.

[1] S. Gianfaldoni, R. Gianfaldoni, U. Wollina, J. Lotti, G. Tchernev, and T. Lotti, An overview on radiotherapy: From its history to its current applications in dermatology, Open Access Macedonian J. Med. Sci. 5, 521 (2017).

[2] J. D. Tward, C. J. Anker, D. K. Gaffney, and G. M. Bowen. Radiation therapy and skin cancer, Mod. Practices Radiat. Therapy 207, 46 (2012).

[3] D. De Ruysscher, C. Faivre-Finn, U. Nestle, C. W. Hurkmans, C. Le Péchoux, A. Price, and S. Senan, European Organisation for Research and Treatment of Cancer recommendations for planning and delivery of high-dose, high-precision radiotherapy for lung cancer, J. Clin. Oncol. 28, 5301 (2010).

[4] C. M. Nutting, J. P. Morden, K. J. Harrington, T. G. Urbano, S. A. Bhide, C. Clark et al., Parotid-sparing intensity modulated versus conventional radiotherapy in head and neck cancer (PARSPORT): A phase 3 multicenter randomized controlled trial, Lancet Oncol. 12, 127 (2011).

[5] M. Yang and R. Timmerman, Stereotactic ablative radiotherapy uncertainties: Delineation, setup and motion, Semin. Radiat. Oncol. 28, 207 (2018).

[6] J. Bourhis, J. Overgaard, H. Audry, K. K. Ang, M. Saunders, J. Bernier et al., Hyperfractionated or accelerated radiotherapy in head and neck cancer: A meta-analysis, Lancet 368, 843 (2006).

[7] V. Favaudon, L. Caplier, V. Monceau, F. Pouzoulet, M. Sayarath, C. Fouillade, M. F. Poupon, I. Brito, P. Hupé, J. Bourhis, and J. Hall, Ultrahigh dose-rate FLASH irradiation increases the differential response between normal and tumor tissue in mice, Sci. Transl. Med. 6, 245 (2014).

[8] K. J. Harrington, Ultrahigh dose-rate radiotherapy: Next steps for FLASH-RT, Clin. Cancer Res. 25, 3 (2018).

[9] M. C. Vozenin, P. De Fornel, K. Petersson, V. Favaudon, M. Jaccard, J. F. Germond, B. Petit, M. Burki, G. Ferrand, D. Patin, and H. Bouchaab, The advantage of FLASH 
radiotherapy confirmed in mini-pig and cat-cancer patients, Clin. Cancer Res. 25, 35 (2019).

[10] M. C. Vozenin, J. H. Hendry, and C. L. Limoli, Biological benefits of ultrahigh dose-rate FLASH radiotherapy: Sleeping beauty awoken, Clin. Oncol. 31, 407 (2019).

[11] M. C. Vozenin, M. Baumann, R. P. Coppes, and J. Bourhis, FLASH radiotherapy International Workshop, Radiotherapy and oncology : Journal of the European Society for Therapeutic Radiology and Oncology 139, 1 (2019).

[12] S. Hornsey and T. Alper, Unexpected dose-rate effect in the killing of mice by radiation. Nature (London) 210, 212 (1966).

[13] J. H. Hendry, J. V. Moore, B. W. Hodgson, and J. P. Keene, The constant low oxygen concentration in all the target cells for mouse tail radionecrosis, Radiat. Res. 92, 172 (1982).

[14] J. Bourhis, P. Montay-Gruel, P. G. Jorge, C. Bailat, B. Petit, J. Ollivier, W. Jeanneret-Sozzi, M. Ozsahin, F. Bochud, R. Moeckli, and J. F. Germond, Clinical translation of FLASH radiotherapy: Why and how?, Radiotherapy Oncol. 139, 11 (2019).

[15] A. Schüller, S. Heinrich, C. Fouillade, A. Subiel, L. De Marzi, F. Romano, P. Peier, M. .1, C. Fleta, R. Kranzer et al., The European Joint Research Project UHDpulseMetrology for advanced radiotherapy using particle beams with ultrahigh pulse dose rates, Phys. Med. 80, 134 (2020).

[16] T. Freeman, FLASH radiotherapy: From preclinical promise to the first human treatment. AAPM Annual Meeting, Phys. World (2019), https://physicsworld. com/a/flashradiotherapy-from-preclinical-promise-to-the-firsthumantreatment/.

[17] M. Durante et al., Commentary-Faster and safer? FLASH ultrahigh dose rate in radiotherapy, Br. J. Radiol. 91, 20170628 (2018).

[18] N. M. Esplen, M. S. Mendonca, and M. Bazalova-Carter, Physics and biology of ultrahigh dose-rate (FLASH) radiotherapy: A topical review, Phys. Med. Biol. (2020).

[19] P. Montay-Gruel, K. Petersson, M. Jaccard, G. Boivin, J. F. Germond, B. Petit, R. Doenlen, V. Favaudon, F. Bochud, C. Bailat, and J. Bourhis, Irradiation in a flash: Unique sparing of memory in mice after whole brain irradiation with dose rates above $100 \mathrm{~Gy} / \mathrm{s}$, Radiotherapy Oncol. 124, 365 (2017).

[20] P. Montay-Gruel, M. M. Acharya, K. Petersson, L. Alikhani, C. Yakkala, B. D. Allen, J. Ollivier, B. Petit, P. G. Jorge, A. R. Syage, and T. A. Nguyen, Long-term neurocognitive benefits of FLASH radiotherapy driven by reduced reactive oxygen species, Proc. Natl. Acad. Sci. U.S.A. 116, 10943 (2019).

[21] J. Bourhis, W. J. Sozzi, P. G. Jorge, O. Gaide, C. Bailat, F. Duclos, D. Patin, M. Ozsahin, F. Bochud, J. F. Germond, and R. Moeckli, Treatment of a first patient with FLASHradiotherapy, Radiotherapy Oncol. 139, 18 (2019).

[22] EN 60601-2-1:2020 Medical electrical equipment-Part 2-1: Particular requirements for the basic safety and essential performance of electron accelerators in the range 1 to $50 \mathrm{MeV}$.

[23] IEC 60976:2007 Medical electrical equipmentMedical electron accelerators-Functional performance characteristics.
[24] IEC 60977, Medical electrical equipment-Medical electron accelerators-Guidelines for functional performance characteristics.

[25] V. Favaudon, J.-M. Lentz, S. Heinrich, A. Patriarca, L. de Marzi, C. Fouillade, and M. Dutreix, Time-resolved dosimetry of pulsed electron beams in very high dose-rate, FLASH irradiation for radiotherapy preclinical studies, Nucl. Instrum. Methods Phys. Res., Sect. A 944, 162537 (2019).

[26] E. Schüler, S. Trovati, G. King, F. Lartey, M. Rafat, M. Villegas, A. J. Praxel, B. W. Loo, Jr., and P. G. Maxim, Experimental platform for ultrahigh dose-rate FLASH irradiation of small animals using a clinical linear accelerator, Int. J. Radiat. Oncol. Biol. Phys. 97, 195 (2017).

[27] K. Petersson, M. Jaccard, J. F. Germond, T. Buchillier, F. Bochud, J. Bourhis, M. C. Vozenin, and C. Bailat, High dose-per-pulse electron beam dosimetry. A model to correct for the ion recombination in the advanced Markus ionization chamber, Med. Phys. 44, 1157 (2017).

[28] M. Lempart, B. Blad, G. Adrian, S. Bäck, T. Knöös, C. Ceberg, and K. Petersson, Modifying a clinical linear accelerator for delivery of ultrahigh dose-rate irradiation, Radiotherapy Oncol. 139, 40 (2019).

[29] B. P. Venkatesulu, A. Sharma, J. M. Pollard-Larkin, R. Sadagopan, J. Symons, S. Neri, P. K. Singh, R. Tailor, S. H. Lin, and S. Krishnan, Ultrahigh dose-rate (35 Gy/ sec) radiation does not spare the normal tissue in cardiac and splenic models of lymphopenia and gastrointestinal syndrome, Sci. Rep. 9, 17180 (2019).

[30] G. Felici, P. Barca, S. Barone, E. Bortoli, R. Borgheresi, S. De Stefano, M. Di Francesco, L. Grasso, S. Linsalata, D. Marfisi, and M. Pacitti, Transforming an IORT linac into a FLASH research machine: Procedure and dosimetric characterization, Front. Phys. 8, 374 (2020).

[31] M. Rahman, M. R. Ashraf, R. Zhang, P. Bruza, C. A. Dexter, L. Thompson, X. Cao, B. B. Williams, P. J. Hoopes, B. W. Pogue, and D. J. Gladstone, Electron FLASH delivery at treatment room isocenter for efficient reversible conversion of a clinical linac, Int. J. Radiat. Oncol. Biol. Phys. (2021).

[32] P. Lansonneur, V. Favaudon, S. Heinrich, C. Fouillade, P. Verrelle, and L. De Marzi, Simulation and experimental validation of a prototype electron beam linear accelerator for preclinical studies, Phys. Med. 60, 50 (2019).

[33] M. McManus, F. Romano, N. D. Lee, W. Farabolini, A. Gilardi, G. Royle, H. Palmans, and A. Subiel, The challenge of ionization chamber dosimetry in ultrashort pulsed high dose rate very high energy electron beams, Sci. Rep. 10, 1 (2020).

[34] F. Di Martino, P. Barca, S. Barone, E. Bortoli, R. Borgheresi, S. De Stefano, M. Di Francesco, L. Faillace, L. Giuliano, L. Grasso, and S. Linsalata, Corrigendum: FLASH radiotherapy with electrons: Issues related to the production, monitoring, and dosimetric characterization of the beam, Front. Phys. 8, 630534 (2021).

[35] F. Di Martino, M. Giannelli, A. C. Traino, and M. Lazzeri, Ion recombination correction for very high dose-per-pulse high-energy electron beams: ksat evaluation for very high dose-per-pulse electron beams, Med. Phys. 32, 2204 (2005). 
[36] http://egun-igun.com/

[37] https://www.3ds.com/

[38] https://www.teledyne-e2v.com/

[39] T. P. Wangler, RF Linear Accelerators (John Wiley \& Sons, New York, 2008).

[40] http://tstep.lmytechnology.com/TSTEP.html

[41] https://laacg.lanl.gov/laacg/services/services.phtml

[42] M. Jaccard et al., High dose-per-pulse electron beam dosimetry: Commissioning of the Oriatron eRT6 prototype linear accelerator for preclinical use, Med. Phys. 45, 863 (2018).

[43] G. P. Jorge et al., Dosimetric and preparation procedures for irradiating biological models with pulsed electron beam at ultrahigh dose rate, Radiotherapy and oncology : Journal of the European Society for Therapeutic Radiology and Oncology 139, 34 (2019).

[44] Gafchromic ${ }^{\mathrm{TM}}$ radiotherapy films, available online at https:// www.ashland.com/industries/medical/medical-radiationdosimetry/gafchromic-radiotherapy-films

[45] G. A. P. Cirrone, G. Petringa, B. M. Cagni, G. Cuttone, G. F. Fustaino, M. Guarrera et al., Use of radiochromic films for the absolute dose evaluation in high dose-rate proton beams, J Inst. 15, C04029 (2020).

[46] FilmQA Pro ${ }^{\mathrm{TM}}$ software, https://www.ashland.com/ industries/medical/medical-radiation-dosimetry/film-qapro-software 